\title{
High resolution frequency selective photochemistry of phycobilisomes at cryogenic temperatures
}

\author{
W. Köhler and J. Friedrich \\ Physikalisches Institut und Bayreuther Institut für Makromolekülforschung, Universität Bayreuth. \\ D-8580 Bayreuth, West Germany \\ R. Fischer and $H$. Scheer \\ Botanisches Institut, Universität München, D-8000 München 19, West Germany
}

(Received 26 January 1988; accepted 28 March 1988)

\begin{abstract}
Highly frequency selective photochemistry at cryogenic temperatures is used to gain information on the excited state dynamics of large biomolecular aggregates, the phycobilisomes from the blue-green alga (cyanobacterium) Masticogladus laminosus. In particular, we show that in spite of the well organized structure of these aggregates disorder on a microscopic level dominates the optical spectra. The hole burning reaction in the resonantly excited chromophores is most probably due to a conformational change in the neighborhood of the chromophore. From the widths of the holes energy transfer times between different pigments on the order of $16 \mathrm{ps}$ are determined. These transfer times are independent of the excitation energy.
\end{abstract}

\section{INTRODUCTION}

Proteins and glasses have many features in common, yet they are very distinct, too. ${ }^{1}$ It seems that the common features show up preferentially at low temperatures: Their specific heat is linear in temperature, they show microwave absorption $^{2-4}$ and nonexponential relaxation features. ${ }^{5}$ These properties are characteristic for the presence of disorder on a microscopic scale. As to the optical properties disorder is most evident in a large inhomogeneous line broadening of the absorption and fluorescence bands, which is on the order of several hundred wave numbers. ${ }^{6,7}$ On the other hand, proteins have on a larger scale a very well defined structure, which is necessary for the correct functioning. ${ }^{1}$ Disorder on a microscopic level reflects the fact that the system under consideration has a huge conformational space. Upon cooling it may be trapped at some point in this space. ${ }^{8}$ In case of chromoproteins it is often possible to select a subensemble of molecules out of the total inhomogeneous band, by exciting the chromophore with a narrow bandwidth laser., ${ }^{9,10}$ The subensemble is made up of those molecules which happen to have their chromophore absorption at the laser frequency. If the excited chromophore is photoreactive, the excited molecules may be transformed to some photoproduct with a different absorption energy. In this case a dip may eventually appear in the spectrum which may show the characteristic features of the homogeneous line shape. ${ }^{6,7}$ In this case the coherence lifetime $T_{2}$ can be determined from the width of the dip. This technique is called photochemical hole burning. However, hole burning also occurs very often even in case the excited chromophore undergoes no photoreaction at all. ${ }^{11-13}$ In this case optical excitation enables a transfer from one point in the conformation space to another which is most probably mediated by the optical energy which is nonradiatively released into the environment of the chromophore. This type of frequency selective photoreactions is called "photophysical."

In the present paper we demonstrate that narrow spectral holes can be burnt into the absorption band of highly organized chromoproteins. The samples investigated are phycobilisomes of the blue-green alga (cyanobacterium) Masticogladus laminosus. The phycobilisomes (PBS) are highly organized supramolecular aggregates which act as photosynthetic antenna pigments. ${ }^{14-18}$ They are built from discs, which contain trimers (heterohexamers) of biliproteins, e.g., phycoerythrocyanin (PEC), phycocyanin (PC), and allophycocyanin (APC), and the so-called linker polypeptides. For PC it was shown that the chromophores are arranged around a threefold symmetry axis. ${ }^{19}$ The PEC and PC discs form rods which group in a rather symmetric fashion around the APC core. Hence, the PBS have a well ordered structure at least on scales larger than a few angstroms. Energy transfer occurs along the rods to the core, from PEC to PC to APC.

In case the energy transfer times are fast compared to the pure dephasing time, the width of the hole should be dominated by energy transfer processes. In this paper we present a series of hole burning experiments at various positions in the visible range of the phycobilisome absorption in order to get information on the related energy transfer times. Due to the complex dynamics of the PBS it is a rather complicated procedure to get information on energy transfer times from time resolved experiments. ${ }^{20}$ Hole burning, on the other hand, is a very direct method which brings about additional information.

\section{MATERIALS AND METHODS}

\section{Preparation of samples}

Masticogladus laminosus was grown in $300 \ell$ batch cultures at the Cesellschaft für Biotechnologische Forschung, Stöckheim. Cells were stored deeply frozen until used. The PBS were isolated according to a procedure described by Nies and Wehrmeyer. ${ }^{21}$ The $p H$ of the buffer system was 6 . To obtain a high level of purification the sucrose gradient centrifugation was repeated twice. In order to get a good quality low temperature glass the PBS were dialized against 
a sucrose saturated potassium phosphate buffer $(0.9 \mathrm{M}, p \mathrm{H}$ 6).

\section{Hole burning spectroscopy}

Holes were burnt with an $\mathrm{Ar}^{+}$-laser pumped dye laser with a bandwidth of $10^{-4} \mathrm{~cm}^{-1}$. The power density was 50 $\mu \mathrm{W} / \mathrm{cm}^{2}$. The holes were recorded as a function of burning fluence. The "homogeneous" linewidth was then obtained by extrapolation to fluence zero. This procedure ensures that the measured hole width is free of photochemical saturation broadening. The depth of the holes was kept rather low to allow for a linear extrapolation (see, e.g., Ref. 6).

The holes were detected either in fluorescence or in transmission by scanning the burning laser or a high resolution $\left(0.2 \mathrm{~cm}^{-1}\right)$ monochromator. The results did not show any dependence on the detection procedure. Most of the experiments were performed at $4 \mathrm{~K}$.

\section{RESULTS AND DISCUSSION}

\section{Absorption spectrum}

The low temperature ( $4 \mathrm{~K}$ ) absorption spectrum of PBS is shown in Figs. 1 (together with the $\triangle$ OD spectrum) and 3 (together with the fluorescence spectrum). The fluorescence spectrum is evidence that energy transfer from PEC and $\mathrm{PC}$ to the terminal APC chromophores is retained in the low temperature sample. ${ }^{22-24}$ There are several prominent peaks. The $6350 \AA$ band originates from low lying PC chromophores, whereas the $6520 \AA$ band is related to APC. The broad features around $6100 \AA$ correspond to the absorption of high-lying PC and low-lying PEC chromophores. The weak shoulders around $6700 \AA$ may originate from the terminal chromophores, allophycocyanin $\mathrm{B}^{25}$ or the $75 \mathrm{kDa}$ polypeptide. ${ }^{26}$ One striking feature of the absorption spectrum is the fact that all bands show a pronounced inhomogeneous broadening on the order of $400 \mathrm{~cm}^{-1}$, comparable to dye molecules in glasses and polymers, which is indicative of the presence of disorder on a microscopic scale, despite the fact that the PBS have a well-organized structure. Since

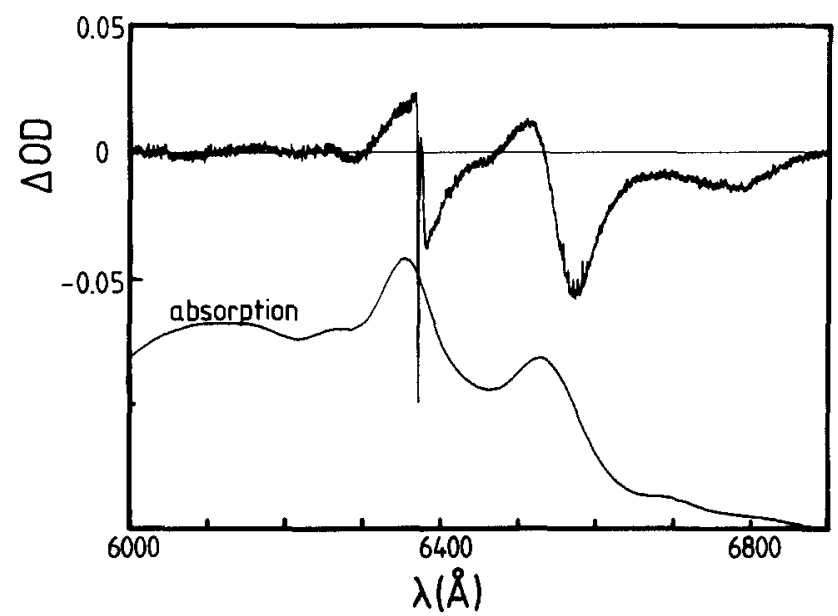

FIG. 1. Low temperature absorption ( $4 \mathrm{~K}$ ) and $\triangle O D$ spectrum of phycobilisomes of Mastigocladus laminosus. Laser excitation was carried out at $6380 \AA$, as documented by the sharp zero phonon hole. some of the chromophores are well shielded from the solvent (e.g., not accessible to the hydrophobic reagent, $\mathbf{P C M S}^{27}$ ), we conclude that the disorder is not due to the surrounding solvent molecules but is, instead, due to intrinsic disorder of the protein. The term "protein" used throughout is meant to include the polypeptide, the chromophores, internal water, and water bound to the surface.

\section{Frequency selective laser photochemistry}

The change in optical density of the PBS absorption upon laser irradiation into the PC absorption around $6370 \AA$ is shown in Fig. 1.

$\Delta O D>0$ indicates those spectral ranges where photoproduct appears, whereas $\Delta \mathrm{OD}<0$ indicates regions where educt has been removed. The sharp zero phonon hole very clearly reflects the fact that frequency selective phototransformation processes occur. The broad phonon side hole adjacent to the zero phonon hole on the long wavelength side shows that a considerable amount of phototransformation takes place via phonon excitations. Figure 1 shows a variety of interesting features:

(i) In the resonantly excited PC-chromophore, the photoproduct appears in the immediate neighborhood of the burning frequency. Its spectral distribution seems to be confined to the inhomogeneous absorption band,

(ii) there is no frequency selective photochemistry in the nonresonantly excited chromophores [APC, terminal emitter(s) ],

(iii) phototransformation processes in the APC peak take preferential place at the red edge of the band, and (iv) there is a net loss of absorption in the long wavelength range from which we conclude that either the product absorbs well outside the spectral range investigated or its oscillator strength is much less than that of the educt.

From observation (i) we conclude that the photoreaction in the resonantly excited chromophore (PC) is most likely photophysical in nature. This means, that it is not the chromophore itself, which is photochemically changed but it is rather its immediate proteinaceous environment. This could, for instance, be due to a slight change of the relative orientation of the chromophore in its solvent cage, as was shown to be the dominant reaction mechanism for tetracene in alcohol glass. ${ }^{13}$ Of course, simple conformational changes in the protein structure around the selected chromophore are also candidates for possible reactions. Recently, we showed that in PBS the distribution of barriers which stabilize the product against the educt state is similar to glasses and polymers. However, unlike those materials, proteins show, in addition, discrete features superimposed the smooth glass-like distribution. ${ }^{8,28}$

Observation (ii) is very characteristic for energy transfer systems. Since in PBS there is fast and efficient energy transfer from PC to APC and the terminal emitter, ${ }^{14-18}$ it is obvious that most of the energy absorbed by the PC is transferred to the lower energy acceptor pigments (see Fig. 3). Hence, photoreactive processes may not only occur in the resonantly excited chromophore, but also in the spectrally shifted acceptor states (APC, terminal emitter). This is 
clearly shown in Fig. 1. The loss of frequency selectivity is indicative of the fact that there is no energy correlation between the PC donor and the acceptor states. ${ }^{29}$ In other words, looking at an individual PBS aggregate, the fluctuation of the energy splitting between PC and, say APC, may have any magnitude which is, roughly speaking, of similar size as the inhomogeneous bandwidths. A loss of energy correlation between two different electronic states is a very characteristic feature of glass-like disorder. ${ }^{30-32}$

A closer look to Fig. 1 shows, however, that it is not the total APC band which is bleached but, instead, it is preferentially the red edge [observation (iii)]. We believe that this phenomenon is due to the fact that there is not only energy transfer between different pigments (e.g., PC to APC) but also between different chromophores in one pigment. This transfer is the so-called $s$ (sensitizing) to $f$ (fluorescing) transfer, and is assumed to be extremely fast, namely on the order of a few picoseconds. ${ }^{22-24,33}$ Hence, at low temperatures, population is piled up preferentially in the $f$ chromophores, which make up the red edge of the band. From there the photoreaction proceeds. These arguments are in line with earlier experiments on C-PC-trimers, which showed that the hole burning yield was much higher within the red edge of the visible band and decreased very fast for higher energies. ${ }^{29}$

Observation (iv) is not yet understood. We could not find any appreciable amount of photoproduct which could account for the large area burnt in the range between 6550 and $6900 \AA$. The most likely explanation is a change in chromophore conformation and/or the surrounding charge distribution. The biliprotein chromophores are known for their high sensitivity in both, band position and oscillator strength, upon such changes. ${ }^{14,15,27,34,35}$ It cannot be excluded, however, that the reaction would change the chromophore in a way that its absorption would be no longer within the spectral range investigated.

\section{Homogeneous linewidth and energy transfer times}

It is well known from basic quantum mechanics that the homogeneous linewidth $\Delta \omega$ of a transition is related to a phase relaxation time $T_{2}$ :

$$
\Delta \omega=1 / T_{2} .
$$

$T_{2}$ itself consists of two contributions, namely an energy relaxation time $T_{1}$ and a pure dephasing time $T_{2}^{*}$,

$$
1 / T_{2}=1 / 2 T_{1}+1 / T_{2}^{*} \text {. }
$$

$T_{2}^{*}$ is a measure of how fast the phase coherence of a transition decays due to energy conserving dynamical processes like electron-phonon scattering - or resonant energy transfer processes (see, for instance, Ref. 6). $T_{2}^{*}$ depends on temperature. In disordered solids this dependence is rather moderate and is, in many cases, well described by a power law with an exponent on the order of 1 to $1.5 .^{36}$ In case $T_{2}^{*}$ is small compared to $1 / 2 T_{1}$, the excited state lifetime $T_{1}$ can be determined from linewidth data. In this case, however, one has to make sure that relation (1) really holds. Disordered systems may undergo structural relaxation processes which may lead to a broadening of the hole on time scales much longer than $T_{1}$. This kind of broadening is called spectral diffusion. ${ }^{37.38}$ Though we did not do any time dependent hole burning experiments in proteins we rule out large contributions to the holewidths from spectral diffusion processes. Figure 2 shows that this conclusion is reasonable. We compare typical hole traces (which are close to their homogeneous shapes) of isolated PC trimers, with PC embedded in intact PBS. There is a striking broadening of the width in going from the isolated chromoprotein to the supramolecular aggregate. We stated above that it is only the low lying $f$ chromophores which are effectively burnt. In the isolated chromoprotein the width of the transition to the $f$ chromophore seems, at $4 \mathrm{~K}$, essentially be determined by phase relaxation processes, which occur on a time scale of $100 \mathrm{ps}$ or so. At $1.5 \mathrm{~K}$ the width in C-PC is only by a factor of 4 off the lifetime limited value which is on the order of $0.01 \mathrm{~cm}^{-1}$ ( = $1 \mathrm{~ns}) \cdot{ }^{22-24}$ In the PBS aggregates the $f$ chromophores of $\mathrm{PC}$ are no longer the emitters, but transfer energy to lower acceptors. Their lifetime should then be dominated by energy transfer processes. We attribute the large width as being caused to a large extent by these additional relaxation processes. From the observed hole width of $0.34 \mathrm{~cm}^{-1}$ we calculate a pigment to pigment transfer time on the order of $16 \mathrm{ps}$. This number should be considered as a lower limit because, at $4 \mathrm{~K}$, there still seems to be some temperature dependent contributions to the width which could originate from other line broadening processes. This number fits into the kinetic scheme of PBS known from time resolved pump-probe and fluorescence experiments. Cillbro et al. found for the PC to APC transfer in PBS from the mutant AN 112 of Synechococcus 6301 time constants on the order of $40-50$ ps..$^{39,40}$ Most interestingly, a similar time constant was found from APC excited fluorescence decay measurements. In this case, it was assigned to the energy transfer from the APC to the terminal chromophores.

Figure 3 shows a survey of all our hole burning experiments on PBS. Shown are the spectral positions where hole burning was performed. As is obvious from the figure, the hole width does not significantly change as a function of frequency, a result which seems to be in line with the time resolved experiments. We have, on the other hand, to con-

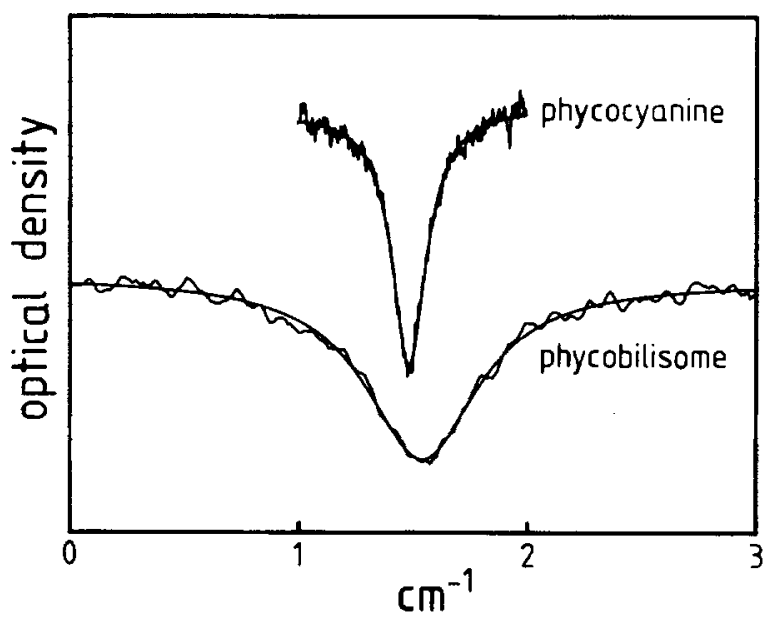

FIG. 2. Hole traces of isolated phycocyanin trimers and phycobilisomes for low burning fluences. 


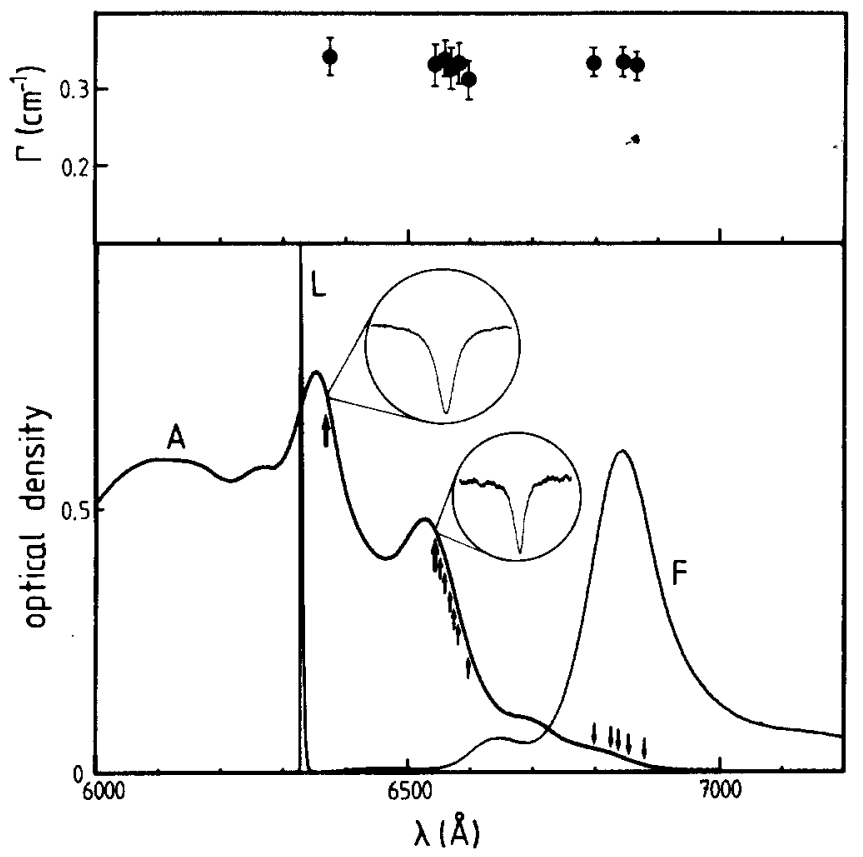

FIG. 3. Absorption and fluorescence spectrum of phycobilisomes. Marked positions indicate hole burning frequencies. Two typical hole traces are shown. $L$ marks the laser line for fluorescence excitation. The upper part of the figure shows the hole width, extrapolated to zero burning fluence, as a function of excitation wavelength.

clude that even irradiating as far as $6780 \AA$ in the red we still do not burn the fluorescing chromophore of the PBS aggregate. Instead, the linewidths still seems to be limited by energy transfer processes.

\section{SUMMARY}

An example is given on how frequency selective photochemistry can be used to gain information on dynamic processes of large biomolecules. We found that in PBS, though they have a highly organized structure, the spectral properties are still dominated by local disorder. There is a large inhomogeneous broadening and a loss of correlation of electronic energy. The hole width is influenced to a considerable extent by energy transfer processes. From the measured width a lower limit for the pigment to pigment transfer time was determined. It is on the order of $16 \mathrm{ps}$ and is independent on the excitation wavelength. These results are corroborated by the fact that in isolated PC the hole width is much smaller than for the same pigment in the intact phycobilisomes. The hole burning reaction in the resonantly excited chromophores is most probably due to a photophysical process which corresponds to a conformational change in the proteinaceous environment (polypeptide chromophore-bound water ensemble) of the excited chromophore. However, the nature of the reaction in the chromophores excited by energy transfer processes is not yet very clear.

\section{ACKNOWLEDGMENTS}

We are indebted to G. Reng (Gesellschaft für Biotechnologische Forschung, Stöckheim) for mass culture of $M$. laminosus. We thank the Deutsche Forschungsgemeinschaft for financial support (SFB 213, Bayreuth, SFB 143, and Forschergruppe "Pflanzliche Tetrapyrrole," München).

'H. Frauenfelder, Helv. Phys. Acta 57, 165 (1984).

${ }^{2}$ G. P. Singh, F. Parak, S. Hunklinger, and K. Dransfeld, Phys. Rev. Lett. 47, 685 (1981).

${ }^{3}$ G. P. Singh, H. J. Schink, H. von Löhneysen, F. Parak, and S. Hunklinger, Z. Phys. B 55, 23 (1984).

${ }^{4} I .-S$. Yang and A. C. Anderson, Phys. Rev. B 34, 2942 (1986).

${ }^{5}$ A. Ansari, J. Berendzen, S. F. Bowne, H. Frauenfelder, J. E. T. Iben, T. B. Sauke, E. Shyamsunder, and R. D. Young, Proc. Natl. Acad. Sci. U.S.A. 82, 5000 (1985).

${ }^{6}$ J. Friedrich and D. Haarer, Angew. Chem. 96, 96 (1984); Int. Ed. Engl. 23, 113 (1984).

${ }^{7}$ J. M. Hayes, R. Jankowiak, and G. J. Small, in Persistent Spectral Hole Burning: Science and Application, edited by W. E. Moerner (Springer, New York, 1987).

${ }^{8}$ W. Köhler, J. Friedrich, and H. Scheer, Phys. Rev. A 37, 660 (1988).

${ }^{9} \mathrm{~J}$. Friedrich, H. Scheer, B. Zickendraht-Wendelstadt, and D. Haarer, J. Chem. Phys. 74, 2260 (1981); J. Am. Chem. Soc. 103, 1030 (1981).

${ }^{10}$ I. Renge, K. Mauring, and R. Aavarma, Biochim. Biophys. Acta 766, 501 (1984).

"B. M. Kharlamov, R. I. Personov, and L. A. Bykovskaya, Opt. Commun. 12, 191 (1974).

${ }^{12}$ J. M. Hayes and G. J. Small, Chem. Phys. 27, 151 (1978).

${ }^{13}$ W. Köhler, J. Meiler, and J. Friedrich, Phys. Rev. B 35, 4031 (1987).

${ }^{14} \mathrm{H}$. Scheer, Angew. Chem. 93, 230 (1981); Int. Ed. Engl. 20, 241 (1981).

${ }^{15}$ R. MacColl and D. Guard-Friar, Phycobiliproteins (Chemical Rubber, Boca Raton, FL, 1987).

${ }^{16}$ E. Gantt, Int. Rev. Cytol. 66, 45 (1980).

${ }^{17}$ A. N. Glazer, Annu. Rev. Biophys. Chem. 14, 47 (1985).

${ }^{18} \mathrm{~W}$. Wehrmeyer, in Proteins and Nucleic Acids in Plant Systematics, edited by U. Jensen and D. E. Fairbrothers (Springer, Berlin, 1983).

${ }^{19}$ T. Schirmer, W. Bode, R. Huber, W. Sidler, and H. Zuber, J. Mol. Biol. 184, 257 (1985).

${ }^{20}$ I. Yamazaki, M. Mimuro, T. Murao, T. Yamazaki, K. Yoshihara, and Y. Fujita, Photochem. Photobiol. 30, 233 (1984).

${ }^{21} M$. Nies and W. Wehrmeyer, Planta 150, 330 (1980).

${ }^{22}$ Many aspects of phycobiliprotein energy transfer are treated in Photosynthetic Light Harvesting Complexes, edited by H. Scheer and S. Schneider, (W. de Gruyter, Berlin, 1988).

${ }^{23} \mathrm{H}$. Scheer, in Photosynthesis III, Encyclopedia of Plant Physiology, New Series, edited by L. A. Staehelin and C. J. Arntzen (Springer, Berlin, 1986), Vol. 19, p. 327.

${ }^{24}$ A. R. Holzwarth, in Topics in Photosynthesis, edited by J. Barber (Elsevier, Amsterdam, 1987).

${ }^{25}$ A. N. Glazer and D. Bryant, Arch. Microbiol. 104, 15 (1975).

${ }^{26}$ T. E. Redlinger and E. Gantt, Plant Physiol. 68, 1375 (1981); see also E. Gantt in Ref. 21, p. 7.

${ }^{27}$ R. Fischer, S. Siebzehnrübl, G. Schmidt, and H. Scheer, in Ref. 20, p. 71. ${ }^{28}$ W. Köhler and J. Friedrich, Phys. Rev. Lett. 59, 2199 (1987).

${ }^{29}$ W. Köhler, J. Friedrich, R. Fischer, and H. Scheer, Chem. Phys. Lett. (in press).

${ }^{30}$ J. Friedrich and D. Haarer, J. Chem. Phys. 79, 1612 (1983).

${ }^{31}$ A. A. Gorokhovskii and J. Kikas, Opt. Commun. 21, 272 (1977).

${ }^{32}$ G. W. Suter, A. J. Kallir, and U. P. Wild, Chimica 37, 413 (1983).

${ }^{33}$ K. Sauer, H. Scheer, and P. Sauer, Photochem. Photobiol. 46, 427 (1987).

${ }^{34}$ H. Scheer, H. Formanek, and S. Schneider, Photochem. Photobiol. 36, 259 (1982).

${ }^{35}$ S. Schneider, C. Scharnagl, M. Dürring, T. Schirmer, and W. Bode, in Ref. 21, p. 483.

${ }^{36}$ H. P. H. Thijssen, R. E. van den Berg, and S. Völker, Chem. Phys. Lett. 97, 295 (1983).

${ }^{37}$ W. Breinl, J. Friedrich, and D. Haarer, J. Chem. Phys. 81, 3915 (1984).

${ }^{38} \mathrm{~J}$. Friedrich and D. Haarer, in Optical Spectroscopy of Glasses, edited by I. Zschokke (Reidel, Dordrecht, 1986), p. 149.

${ }^{39}$ T. Gillbro, A. Sandström, V. Sundström, J. Wendler, and A. R. Holzwarth, Biochim. Biophys. Acta 808, 51 (1985).

${ }^{40}$ A. R. Holzwarth, Picosecond Fluorescence Spectroscopy and Energy Transfer in Photosynthetic Antenna Pigments, edited by J. Barber (Elsevier, Amsterdam, 1987). 